\title{
Current state of the art on tailoring the MXene composition, structure, and surface chemistry
}

Per O A Persson and Johanna Rosén

The self-archived postprint version of this journal article is available at Linköping University Institutional Repository (DiVA):

http://urn.kb.se/resolve?urn=urn:nbn:se:liu:diva-162962

N.B.: When citing this work, cite the original publication.

Persson, P. O A, Rosén, J., (2019), Current state of the art on tailoring the MXene composition, structure, and surface chemistry, Current opinion in solid state \& materials science, 23(6), UNSP 100774. https://doi.org/10.1016/j.cossms.2019.100774

Original publication available at:

https://doi.org/10.1016/j.cossms.2019.100774

Copyright: Elsevier

http://www.elsevier.com/

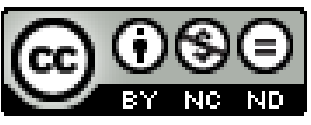




\title{
Current state of the art on tailoring the MXene composition, structure, and surface chemistry
}

Per. O.Å. Persson and Johanna Rosen

Thin Film Physics Division, Department of Physics Chemistry and Biology, Linköping University, 58183 Linköping. Sweden

Corresponding author e-mail: per.persson@liu.se, johanna.rosen@liu.se

\begin{abstract}
MXenes constitute a family of two-dimensional transition metal carbides, carbonitrides and nitrides. Discovered in 2011, the number of MXenes has expanded significantly and more than 20 different MXenes have been synthesized, with many more predicted from theoretical calculations. MXenes constitute an exceptional family of materials based on their availability for elemental alloying and control of surface terminations, which enables synthesis of a range of structures and chemistries. Consequently, the MXenes exhibit an unparalleled potential for tuning of the materials properties for a wide range of applications. At present, MXenes have emerged with astonishing electronic, optical, plasmonic and thermoelectric properties. This has resulted in a global surge of research around a wide variety of applications, including but not limited to energy storage, carbon capture, electromagnetic interference shielding, reinforcement for composites, water filtering, sensors, and photo-, electro- and chemical catalysis etc. In this review, we present the available state of the art tailoring of the MXene properties owing to recent advances in structural ordering and tuning of surface terminations.
\end{abstract}




\section{Introduction}

Transition metal carbides, carbonitrides and nitrides, MXenes, constitute the latest addition to the ever-growing family of two-dimensional (2D) materials.[1,2,3] MXenes were first discovered in 2011 when $\mathrm{Ti}_{3} \mathrm{C}_{2} \mathrm{~T}_{x}$ was reported, and this material has remained the archetype MXene ever since.[1] Currently, in excess of 20 complementary structures have been synthesized with many more predicted from theoretical investigations.[4,5] The MXenes are predominantly synthesized by wet-chemical exfoliation of the parent MAX phases, which follow the general formula $\mathrm{M}_{n+1} \mathrm{AX}_{n}(n=1,2$ or 3$)$, where $\mathrm{M}$ is an early transition metal, $\mathrm{A}$ is a group $\mathrm{A}$ element — mostly groups 13 and 14-, and $\mathrm{X}$ is either C or N. The MAX phases are a group of nanolaminated materials where single A layers separate $\mathrm{M}_{\mathrm{n}+1} \mathrm{X}_{\mathrm{n}}$ slabs.[6] To produce MXene, the MAX phase is chemically etched, resulting in the targeted removal of the A layers, separation of the MX-slabs and their immediate termination by etchant species. Accordingly, the proper MXene description is $M_{\mathrm{n}+1} X_{\mathrm{n}} T_{\mathrm{x}}$, where $T_{x}$ constitute the surface terminating functional atoms or molecules.[7] From the formula, it is evident that MXenes can be tailored through three approaches, including the composition (i.e. the choice of $\mathrm{M}$ and $\mathrm{X}$ elements), the thickness of the sheet ( $n$ ) and the surface terminating species $T_{x}$ (henceforth omitted from formulas for simplicity, unless specifically discussed).

The choice of M and X is primarily governed by the ability to prepare the parent MAX phases and to chemically etch them. It further allows for simple alloying (solid solutions) by diverse elements of both $\mathrm{M}$ and $\mathrm{X}$, and a number of both $\mathrm{M}$ and $\mathrm{X}$ elemental solid solutions have been reported for MAX phases,[8] and for the emerging MXenes.[2] More recently, chemical ordering in MAX phase alloys have also realized chemically ordered MXenes, as shown in fig. 1, allowing new elemental combinations and introduction of elements previously not used for MAX phases.[9] Importantly, so called targeted etching have also created MXenes with ordered vacancies, further expanding the space of attainable properties, summarized below. 
While there is significant progress in tailoring of the MXenes, there are fewer attempts to modify the surface terminations, though these arguably constitute a powerful variable for property tuning. Currently, the MXene preparation dictates that $T_{x}$ constitute species from the etchant, whose ratio can be tailored.[10] Despite several theoretical investigations, non-inherent terminations have remained relatively unexplored experimentally. Only recently, routes for post processing of the MXenes to substitute inherent with non-inherent terminations have been identified and are summarized below.

It should be stressed that the intention of this review is to provide an overview of the current state of the art in terms of how far the MXene community has reached in terms of tailoring the MXene structure, composition, and surface chemistry as well as an indication of where the field is heading. 


\section{MXene structure and composition}

Since the structure and composition of the parent MAX phase is inherited by the MXene, MAX phase research to a large extent governs the progress in MXenes. Apart from random solid solutions on the M site [2], particular attention has been paid in recent years to MAX structures wherein the alloying $\mathrm{M}$ elements are ordered. The first chemically ordered MAX phase alloy, $\left(\mathrm{Cr}_{2 / 3} \mathrm{Ti}_{1 / 3}\right)_{3} \mathrm{AlC}_{2}$, was discovered in 2014.[11] This structure is fundamentally reduced to $M_{3} A X_{2}$, with two $\mathrm{M}$ elements in a 2:1 ratio forming a phase with out-of-plane chemical order through alternating layers composed of one type of $M$ element only. This was soon followed also by out-of-plane ordering in a $M_{4} A X_{3}$ structure with the $\mathrm{M}$ elements in a 2:2 ratio, $\mathrm{Mo}_{2} \mathrm{Ti}_{2} \mathrm{AlC}_{2}$, and the first corresponding out-of-plane MXenes were synthesized,[12] today commonly referred to as $o$-MAX, see Fig. 1 (right schematic) and Fig. 2a-b. The $\mathrm{Mo}_{2} \mathrm{TiC}_{2}, \mathrm{Mo}_{2} \mathrm{Ti}_{2} \mathrm{C}_{3}$, and $\mathrm{Cr}_{2} \mathrm{TiC}_{2}$ MXenes were followed by the discovery of $\mathrm{Mo}_{2} \mathrm{ScC}_{2}$,[13] see fig. 2c-d, wherein the double transition metal content being arranged as inner or outer MXene layers increases the attainable property space. For example, $\mathrm{Ti}_{3} \mathrm{C}_{2} \mathrm{~T}_{x}$ exhibits metal-like behavior, while exchanging the outer Ti layers to obtain the isostructural $\mathrm{Mo}_{2} \mathrm{TiC}_{2} \mathrm{~T}_{\mathrm{x}}$ instead induce semiconductor-like properties.[14,15] Moreover, magnetoresistance measurements of the two materials also show opposite sign, suggesting fundamentally different transport mechanisms. More recently, $o$-MAX phases have been the topic of experimental investigations with respect to for example HER,[16,17] as well as electrical and thermoelectric properties.[18] Furthermore, theoretical studies have explored the electronic,[14,19] magnetic,[20] and catalytic properties.[17] 


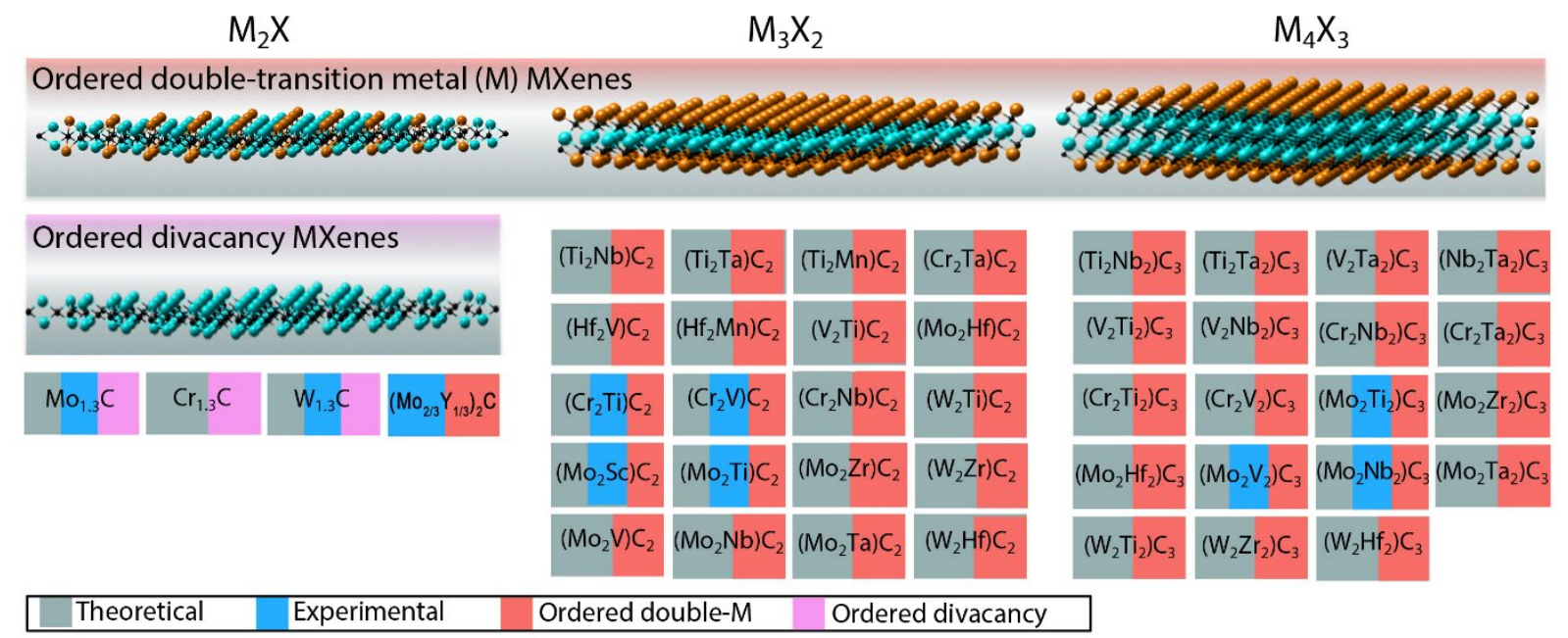

Figure 1. Reported chemically-ordered MXenes from simulations and/or experiments, described by three different general formulas: $M_{2} X, M_{3} X_{2}$ and $M_{4} X_{3}$, where $M$ is an early transition metal and $X$ is carbon and/or nitrogen. Three types of ordering have been found to date; i) out-of-plane chemical order for $M_{3} X_{2}$ and $M_{4} X_{3}$, in which one transition metal occupies the perimeter layers and another fills the central $M$ layers (for example, $\mathrm{Mo}_{2} \mathrm{TiC}_{2}$ and $\mathrm{Mo}_{2} \mathrm{Ti}_{2} \mathrm{C}_{3}$, in which the outer $M$ layers are Mo and the central $M$ layers are Ti), ii) in-plane chemical order for $M_{2} X$, in which one transition metal forms a honeycomb pattern and another occupies the center of the honeycomb (for example $\left(\mathrm{Mo}_{2 / 3} Y_{1 / 3}\right)_{2} \mathrm{C}$, and iii) ordering of $M$-vacancies (as in $M o_{1.33} C$ where the minority $M$ has been selectively etched). Adapted from [9]

In the quest for new chemically ordered double-transition-metal MXenes, Anasori et al. investigated the relative stability of $17 M_{2}{ }_{2} M^{\prime \prime} \mathrm{C}_{2}$ and $15 M^{\prime}{ }_{2} M^{\prime}{ }_{2} \mathrm{C}_{3}$ MXenes from hypothetical $o$-MAX phases with different chemical ordering in the form of fully ordered and partially ordered configurations. [12] Later, Tan et al. explored the structure-stability relationship for eight MXene alloy systems by mapping the degree of ordering vs temperature using high-throughput computation.[21] 
Altogether, these studies suggest that close to 30 ordered double-transition-metal MXenes should be stable with respect to the disordered counterparts. Even though these studies do not take into account the stability of the parent 3D materials likely needed to realize these chemically ordered MXenes, they point towards an extended family of materials with intriguing compositions and properties. With the advancing techniques available for MAX phase synthesis, including stable as well as metastable materials, and an extended range of chemical etching procedures for synthesis of the 2D counterparts, novel future compositions of realized $o$-MAX are expected.
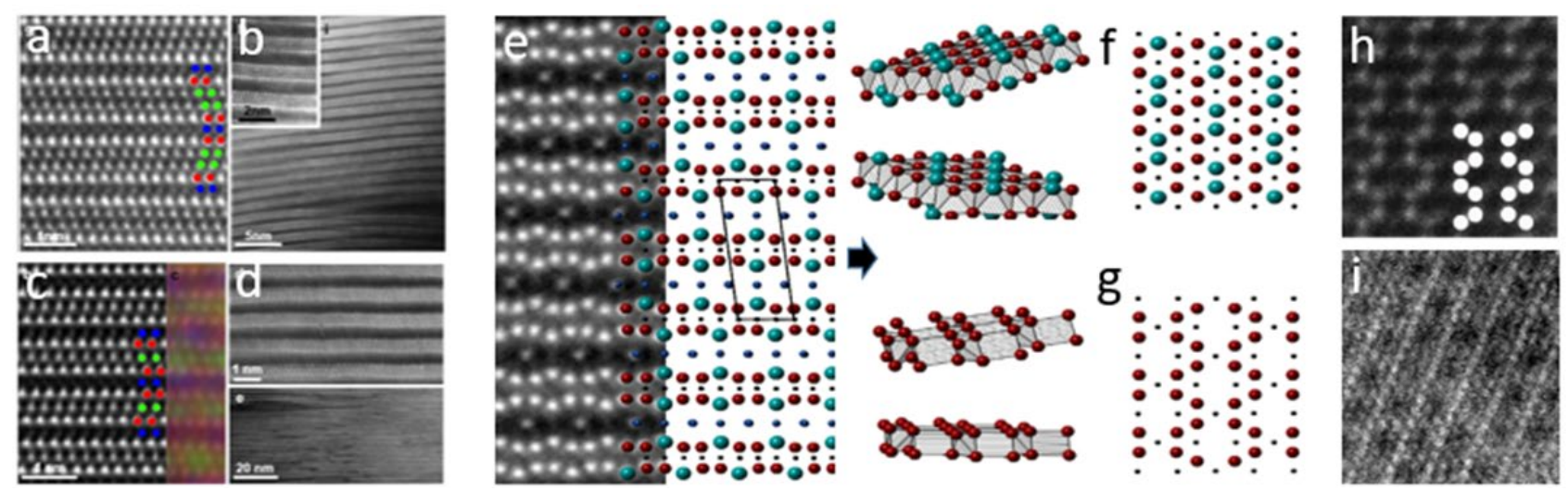

Figure 2: a) $\mathrm{Mo}_{2} \mathrm{Ti}_{2} \mathrm{AlC}_{2} \mathrm{o}-\mathrm{MAX}$ and b) its corresponding $\mathrm{MXene} \mathrm{Mo}_{2} \mathrm{Ti}_{2} \mathrm{C}_{2}$, c) $\mathrm{Mo}_{2} \mathrm{ScAlC}_{2} \mathrm{o}-\mathrm{MAX}$ and d) $\mathrm{Mo}_{2} \mathrm{ScC}_{2}$ MXene with retained chemical order. e) Example of an i-MAX phase, $\left(M_{2 / 3} Y_{1 / 3}\right)_{2} A l C$, which after etching and delamination turns into (schematically) f) a chemically ordered MXene, or g) a MXene with ordered vacancies, also shown in top view (STEM) in h). i) is another example of chemically ordered MXene (top view), including ordering between two Melements as well as vacancies. Adapted from 22, 27]

In 2017, ordering between $M$ elements of a 2:1 ratio was discovered in a $M_{2} A X$ phase, $\left(\mathrm{Mo}_{2 / 3} \mathrm{Sc}_{1 / 3}\right)_{2} \mathrm{AlC},[22]$ though this time with an in-plane chemical arrangement, spurring the notation $i$-MAX.[23] The most characteristic features of these phases is the minority $\mathrm{M}$ element extending from the M-layer towards the A-layer, see fig. 2e), and the A-layer rearranging to form a Kagomélike lattice. The crystal structure of this phase is described by a monoclinic $(C 2 / c)$ structure, as 
opposed to the hexagonal (P63/mmc) structure of traditional MAX phases. To date, more than $20 i$ MAX phases have been discovered (see e.g. reference [24] and references therein) and they all crystallize in one or more of the space group symmetries $C / 2 c(\# 15), C m c m$ (\#63), and C/2m (\#12), close to degenerate in energy according to theoretical simulations. Most of them are based on $\mathrm{A}=\mathrm{Al}$, suggesting that a range of novel MXenes can be synthesized in line with the chemical etching routes used for traditional MAX phases.

Using the first $i$-MAX phase, $\left(\mathrm{Mo}_{2 / 3} \mathrm{Sc}_{1 / 3}\right)_{2} \mathrm{AlC}$, Tao et al. demonstrated chemical removal of not only the Al layer upon etching in $\mathrm{HF}$ or $\mathrm{LiF} / \mathrm{HCl}$, but also selective etching of the minority $M$ element $\mathrm{Sc}$, realizing $\mathrm{Mo}_{1.33} \mathrm{C}$ MXene layers with ordered divacancies, see schematic and top view STEM in fig. 2g-h).[22] A more detailed description of the structure of $i$-MXene from theoretical simulations can be found in [25]. Selective etching of chemically-ordered $i$-MAX phases for synthesis of vacancyordered MXene has been shown for several compositions: Removing $\mathrm{Al}$ and $\mathrm{Sc} / \mathrm{Y}$ from $\left(\mathrm{Mo}_{2 / 3} \mathrm{Sc}_{1 / 3}\right)_{2} \mathrm{AlC}$ and $\left(\mathrm{Mo}_{2 / 3} \mathrm{Y}_{1 / 3}\right)_{2} \mathrm{AlC}$ both produces $\mathrm{Mo}{ }_{1.33} \mathrm{C}$ MXene, while $\left(\mathrm{W}_{2 / 3} \mathrm{Sc}_{1 / 3}\right)_{2} \mathrm{AlC}$ and $\left(\mathrm{W}_{2 / 3} \mathrm{Y}_{1 / 3}\right)_{2} \mathrm{AlC}$ correspondingly yields $\mathrm{W}_{1.33} \mathrm{C}$ MXene.[22,26,27]

The work on $i$-MAX phases has also motivated the concept of "targeted etching", which is detailing the chemical etching procedures to obtain either a vacancy MXene, or a chemically ordered MXene from the same parent material, as shown for $\left(\mathrm{Mo}_{2 / 3} \mathrm{Y}_{1 / 3}\right)_{2} \mathrm{AlC},[27]$ see schematic in Fig. $2 \mathrm{f}$. The tuning potential of $i$-MAX phases as precursors for MXenes is evident when comparing the bond strength of Sc-MX and $\mathrm{Y}-\mathrm{MX}$ in $\left(\mathrm{Mo}_{2 / 3} \mathrm{Sc}_{1 / 3}\right)_{2} \mathrm{AlC}$ and $\left(\mathrm{Mo}_{2 / 3} \mathrm{Y}_{1 / 3}\right)_{2} \mathrm{AlC}$, respectively. Sc, which is more loosely bonded to the MX-layer, can be readily etched from its $i$-MAX phase, while the more strongly bonded Y can be preserved in the MXene upon etching.

Surface terminations are crucial for the properties of MXenes, independent of being of the traditional type or chemically- or vacancy-orederd. The chemical formula from XPS of the vacancy MXene 
$\mathrm{Mo}_{1.33} \mathrm{C}$ obtained from etching $\left(\mathrm{Mo}_{2 / 3} \mathrm{Sc}_{1 / 3}\right)_{2} \mathrm{AlC}$ in $\mathrm{HF}$ followed by $\mathrm{TBAOH}$ intercalation, was reported as $\mathrm{Mo}_{1.2} \mathrm{CO}_{0.7}(\mathrm{OH})_{0.5} \mathrm{~F}_{1.1} \cdot 0.4 \mathrm{H}_{2} \mathrm{O}_{\text {ads }}$, with $\mathrm{F}$ as the primary termination.[25] This stands in contrast to the $\mathrm{Mo}_{2} \mathrm{C}$ MXene without vacancies, with terminations being dominated by $\mathrm{O}$. The difference between the two MXenes are attributed to the different Mo content, where Mo contributes with electrons to both the $\mathrm{C}$ and the terminating species. For $\mathrm{O}$ terminations only, the vacancy MXene have a shortage of electrons, while for $\mathrm{F}$ terminations, with one more electron compared to $\mathrm{O}$, the orbitals can be filled and the structure stabilized.

The exploration of properties of MXenes from $i$-MAX phases are still in its infancy, though the first vacancy MXene, $\mathrm{Mo}_{1.33} \mathrm{C}$, is an excellent electrode material for supercapacitors.[22,28] Pseudocapacitance is the major operative mechanism for a film of the vacancy MXene, as postulated for other MXenes.[29] Utilizing the promising pseudocapacitive properties, Mo1.33 $i$-MXene was also found to be an effective material for removing cations and anions.[30] Furthermore, the most recently reported $\mathrm{W}_{1.33} \mathrm{C} i$-MXene is another promising catalyst for hydrogen evolution reaction.[26] While the initial results are promising, the optimizing potential is large, e.g., tuning the structure and composition of the MXene by changing the etching conditions, and tuning the surface valence states by modifying the surface terminations.

Altogether, both $o$ - and $i$-MAX phases have allowed new elemental combinations and introduction of elements previously not used for MAX phases, such as Sc, Y, and W,[22,26,27] which have led to, e.g., the first W-based MXene.[26] This has vastly expanded the parameter space for future attainable materials properties among MXenes. The ordered vacancy MXenes have also inspired investigations of intentionally introduced disordered vacancies in the MXene sheets. One such example is $\mathrm{Nb}_{1.33} \mathrm{C}$ MXene, which is derived from chemical etching of $\mathrm{Al}$ and $\mathrm{Sc}$ from the parent $\left(\mathrm{Nb}_{2 / 3} \mathrm{Sc}_{1 / 3}\right)_{2} \mathrm{AlC} \mathrm{MAX}$ phase solid solution.[31] 
Even single point defects may prove useful in future applications for MXene. While X site vacancies have been investigated from a theoretical perspective, [32] single vacancies on the $M$ site were reported in the early observations of single sheet $\mathrm{Ti}_{3} \mathrm{C}_{2} \mathrm{~T}_{\mathrm{x}},[33,34]$ and have been investigated theoretically since. Conclusively, the nature of the defect strongly affects both magnetic and electronic properties of the MXene. [35] Vacancies are inferred to originate from an extended or harsh etching process that eventually also affects the MXene sheets in addition to the A-layer. The cumulative effect of multiple point defects further changes the structural prerequisites of the MXene and may produce porous structures. Such MXenes have shown to increase the ion accessibility to active sites. [36] $\mathrm{M}$ site vacancies further present an interesting opportunity as host sites for single atom catalysts such as $\mathrm{Pt}, \mathrm{Ru}, \mathrm{Ir}, \mathrm{Rh}$ and $\mathrm{Pd},[37,38]$ that have proven highly efficient for hydrogen evolution reaction (HER) and for formulation of amines from $\mathrm{CO}_{2}$.

There are opportunities as well as remaining questions, which motivates further investigations. For example, to explore the role of chemical order as well as ordered and disordered vacancies on the materials properties, and to investigate to what extent can we control the formation of vacancies, their concentration, and for which materials. An intriguing example of what the future may bring with respect to control of MXene structure and composition, is the most recently discovered chemical order of a MXene including rare earth (RE) elements, derived from an $i$-MAX phase with the minority element being a $\mathrm{RE}$ metal, $\left(\mathrm{M}_{2 / 3} \mathrm{RE}_{1 / 3}\right)_{2} \mathrm{AlC}$. Instead of forming a vacancy-ordered MXene after removal of $\mathrm{Al}$ and $\mathrm{RE}$, or a chemically ordered MXene equivalent to $\left(\mathrm{M}_{2 / 3} \mathrm{RE} \mathrm{E}_{1 / 3}\right)_{2} \mathrm{C}$ after removal of $\mathrm{Al}$, only selected RE elements are removed together with the Al. A new type of chemical ordering is displayed, evident from Fig. 2i, accentuating the RE atomic distribution, which is organized as continuous lines. The latter example shows the prospects of future tuning of the MXene structure and composition, in the form of controlling point defects, vacancy/atom clustering, and 1D arrays of atoms in a $2 \mathrm{D}$ sheet. This provides a new playground within condensed matter physics, for exploring 
novel materials with outstanding properties, and for providing an expanded test bench for materials modelling. 


\section{Surface chemistry}

During the MAX to MXene etching procedure, the freshly exposed and highly reactive M-element surfaces are immediately functionalized by surface terminating species that originate from the etchant and that are foreign to the parent MAX phase. Accordingly, the MXene formula $M_{n+1} X_{n} T_{x}$ is emphasized to stress the significance of the surface terminations $T_{x}$.

First-principles calculations have in numerous investigations shown the preference for termination of the bare MXene surface. The MXenes gain significant negative formation energy upon surface termination, which indicates the formation of strong bonds between the surface $M$ atoms and the terminating species. $[4,39]$ The terminating species are traditionally inherited from the etchant, which is typically aqueous $\mathrm{HF}$. $\mathrm{Ti}_{3} \mathrm{AlC}_{2} \mathrm{MAX}$ phase immersed in an aqueous solution of $\mathrm{HF}$ was proposed to result in terminated MXene from the following reactions:

$$
\begin{aligned}
& \mathrm{Ti}_{3} \mathrm{AlC}_{2}+3 \mathrm{HF}=\mathrm{AlF}_{3}+3 / 2 \mathrm{H}_{2}+\mathrm{Ti}_{3} \mathrm{C}_{2} \\
& \mathrm{Ti}_{3} \mathrm{C}_{2}+2 \mathrm{H}_{2} \mathrm{O}=\mathrm{Ti}_{3} \mathrm{C}_{2}(\mathrm{OH})_{2}+\mathrm{H}_{2} \\
& T i_{3} \mathrm{C}_{2}+2 \mathrm{HF}=\mathrm{Ti}_{3} \mathrm{C}_{2} \mathrm{~F}_{2}+\mathrm{H}_{2}
\end{aligned}
$$

Based on a c-parameter obtained from XRD analysis and complementary DFT calculations, the surface terminations were proposed to be $\mathrm{OH}$ and/ or F. Subsequent research has concluded that the termination chemistry is more complex, and that surface terminations should additionally include O. $[4,40,41]$ The explanation for the observed surface bound $\mathrm{O}$ was proposed to occur as the surface bound $\mathrm{OH}$ recombine into $\mathrm{H}_{2} \mathrm{O}$ and $\mathrm{O}$ by an equilibrium driven reaction. [42] The relative stability of the $\mathrm{O}, \mathrm{OH}$ and $\mathrm{F}$ (as well as $\mathrm{H}$ ) terminating species has been addressed from both theoretical and experimental considerations. Based on Bader charge analysis and thermodynamic stability analysis it was concluded that the order of stability goes as $\mathrm{Ti}_{3} \mathrm{C}_{2} \mathrm{O}_{2}>\mathrm{Ti}_{3} \mathrm{C}_{2} \mathrm{~F}_{2}>\mathrm{Ti}_{3} \mathrm{C}_{2} \mathrm{OH}_{2}>\mathrm{Ti}_{3} \mathrm{C}_{2} \mathrm{H}_{2} \cdot[39,43]$ 
Recent experimental investigations find little support for $\mathrm{OH}$ terminations and suggest atomic $\mathrm{O}$ as the exclusive O-based termination.[44,45] These findings are supported by a theoretical investigation,[42] which addressed the stability of terminations and found that $\mathrm{OH}$ terminations are intrinsically unstable and a dehydrogenation mechanism is indeed preferred. Consequently, given that $\mathrm{OH}$ is generally considered as an established termination, further investigations on the stability of $\mathrm{OH}$ as a terminating specie are called for.

a)

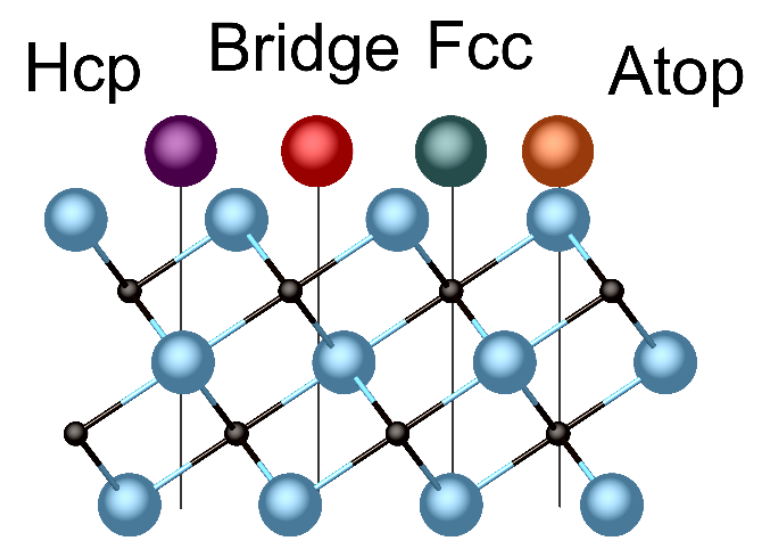

b)

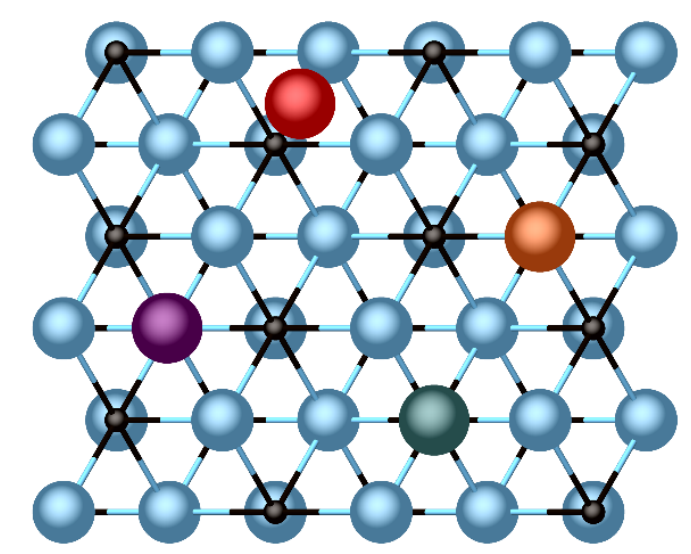

Figure 3: Schematic representation of available low symmetry sites on a $M_{3} X_{2}$ type MXene surface, as viewed in a) cross-section and b) plan-view. The four main sites comprise hcp (purple), bridge (red), fcc (green) and atop (orange), where the vertical position of the terminating species is entirely virtual and changes between species.

The available symmetry sites for MXene surface terminations include the threefold coordinated $f c c$ and $h c p$ sites, as well as the atop position above a surface M element and the bridge position between two top surface M elements, see fig 3. These sites are all energetically stable as shown by theoretical means, however, structure optimization typically transforms the unstable bridge and atop into either fcc or hcp during relaxation. $[4,46]$ Between these two sites, the fcc site has been predicted as the preferred site in numerous studies for native terminations.[4,5,47-52] The fcc site is proposed to be 
favored due to the steric repulsion between the $\mathrm{X}$ element and the terminating specie when positioned in a hcp site.[48] However, some cases have been identified when the terminating specie favors the hcp and the vicinity of an $X$ element in order to attract more electrons.[4,15,19,48] As a general prediction, the relative stability for each site combination (fcc, hcp, mixed) depend on the possible ionic state of the surface transition metal atoms. If the transition metals can assume an oxidation state that provide sufficient electrons to both the $\mathrm{X}$ element and the adsorbed terminating species, the fcc configuration becomes the most stable. Otherwise, hcp or mixed emerge as the most stable configuration.[4]
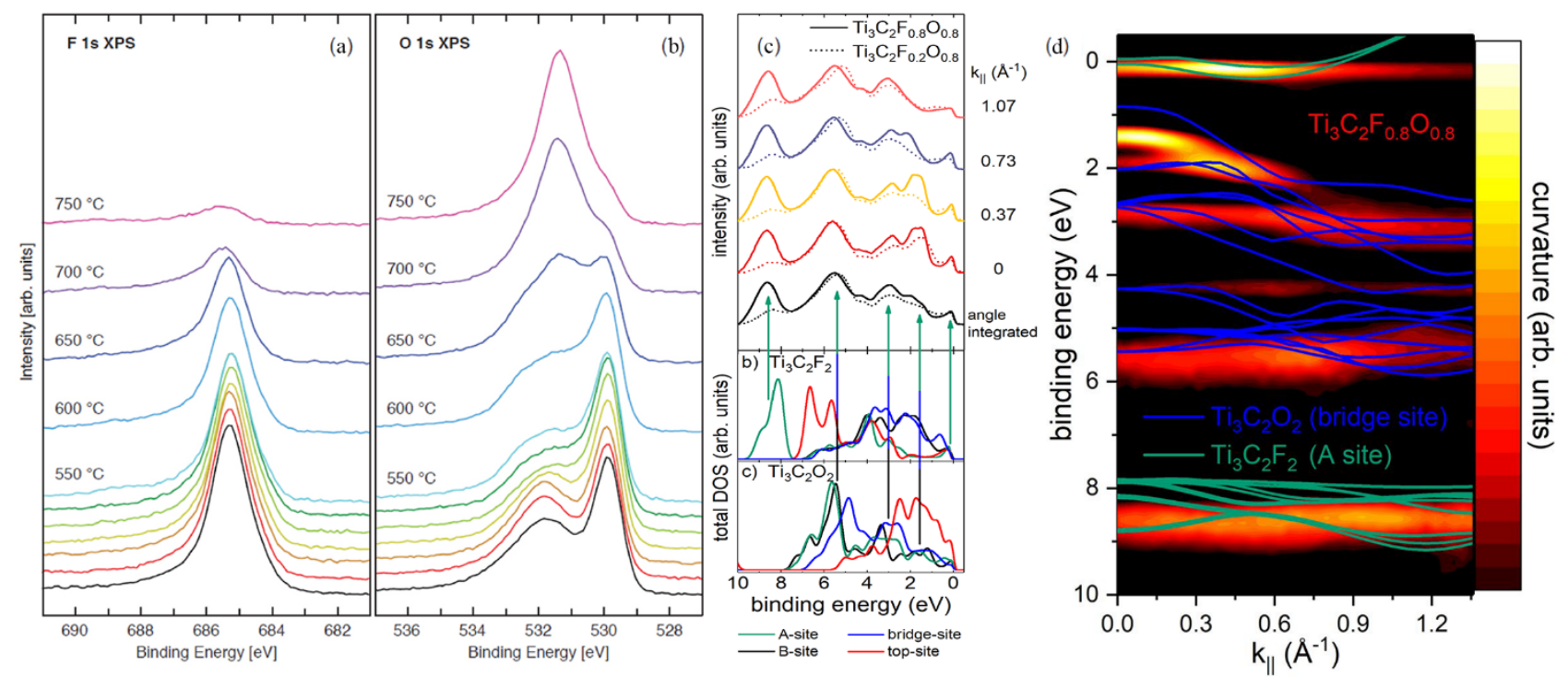

Figure 4: Temperature programmed XPS measurements revealing the temperature dependent behavior of (a) $\mathrm{F} 1 \mathrm{~s}$ and (b) $\mathrm{O}$ 1s. (c) UPS valence band spectra of $\mathrm{Ti}_{3} \mathrm{C}_{2} \mathrm{~F}_{0.8} \mathrm{O}_{0.8}$ (solid lines) and $\mathrm{Ti}_{3} \mathrm{C}_{2} \mathrm{~F}_{0.2} \mathrm{O}_{0.8}$ (dashed lines) for different $k_{\|}$values and angle integrated, with corresponding calculated total DOS for $\mathrm{Ti}_{3} \mathrm{C}_{2} \mathrm{~F}_{2}$ and $\mathrm{Ti}_{3} \mathrm{C}_{2} \mathrm{O}_{2}$ terminations at different adsorption sites. Arrows indicate the correlations between calculations and experimental data. d) Measured ARPES curvature spectrum of $\mathrm{Ti}_{3} \mathrm{C}_{2} \mathrm{~F}_{0.8} \mathrm{O}_{0.8}$ (yellow-red), including a selected part of the calculated band structures of the high-symmetry $\Gamma-\mathrm{K}$ and $\Gamma-\mathrm{M}$ directions for $\mathrm{Ti}_{3} \mathrm{C}_{2} \mathrm{O}_{2}$ with $\mathrm{O}$ adsorbed only on the bridge site (blue) and for $\mathrm{Ti}_{3} \mathrm{C}_{2} \mathrm{~F}_{2}$ with $\mathrm{F}$ adsorbed only on the A site (green). Adapted from [45] and [53] 
The stability and preferred sites for native terminations have been investigated by a range of experimental methods, and the results reveal a complex interplay between $\mathrm{F}$ and $\mathrm{O}$ terminations. A synthesized $\mathrm{Ti}_{3} \mathrm{C}_{2} \mathrm{~T}_{\mathrm{x}}$ thin film, where $\mathrm{T}_{\mathrm{x}}$ was a combination of $\mathrm{O}$ and $\mathrm{F}$, was investigated by temperature programmed X-ray Photoelectron Spectroscopy (XPS).[45] F assumes a single peak, indicating a single preferred site. At increasing temperatures, $\mathrm{F}$ starts to desorb at $\sim 500{ }^{\circ} \mathrm{C}$ until it is desorbed from the surface at around $\sim 750{ }^{\circ} \mathrm{C}$ as shown in figure $4 \mathrm{a}$. $\mathrm{O}$ on the other hand, occupies multiple sites, and as the temperature increases, the $\mathrm{O}$ terminations start to redistribute on the MXene surface. Figure $4 \mathrm{~b}$ shows an initial redistribution already at low temperatures between two sites, and a redistribution to a third site as $\mathrm{F}$ desorbs. The interpretation is evident, that $\mathrm{F}$, due to it's highly electronegative nature, assumes the preferred site immediately upon termination, which $\mathrm{O}$ assumes after F leaves. In situ HRSTEM directly identified this site as fcc. The preference for F on the fcc site was further corroborated by UPS, ARPES and corresponding calculations of the total DOS. [53] This is shown e.g. by the $9 \mathrm{eV}$ non-dispersive feature in figures $4 \mathrm{c}$ and $4 \mathrm{~d}$. For $\mathrm{O}$, the UPS and ARPES measurements showed best agreement for $\mathrm{O}$ at the bridge site for a mixed terminations sample $\left(\mathrm{Ti}_{3} \mathrm{C}_{2} \mathrm{O}_{0.8} \mathrm{~F}_{0.8}\right)$, however, alternative sites could not be ruled out.

Saturation of the MXene surfaces by terminating species occurs when all fcc or all hcp sites are occupied, with one surface terminating specie for each surface M element. At this point the MXene formula becomes $M_{n+1} X_{n} T_{2}$, which is assumed in most investigations. A theoretical investigation addressed the binding energy vs. surface coverage for single species systems on $\mathrm{Ti}_{2} \mathrm{C}$.[15] Fcc sites were first occupied, then additional species were placed in hcp sites to supersaturate the surface. With increasing occupancy of the hcp site, the binding energy per specie increased but remained negative, indicating the possibility for "supersaturation" of the MXene surface. Experimental quantifications predominantly report $\mathrm{x} \leq 2,[54,55]$ but also $\mathrm{x}>2$ was reported.[54] Storage of MXenes at ambient conditions show a slow and gradual oxidation that starts at the F terminated sites, transforming the 
MXenes into metal oxyfluorides and causing a decrease in the amount of $\mathrm{F}$ terminations while increasing the number of $\mathrm{O}$ terminations on the MXene.[54] In particular, for systems in the process of oxidizing to the point of amorphization display $\mathrm{x}>2$.[56] Given the present experimental understanding, the level of saturation of the MXene surfaces by terminating species is not yet understood, and experiments with a tailored surface occupancy and composition are called for. Vice versa, while experimental investigations identify a mix of terminating species and occupancy both above and below $\mathrm{x}=2$, only a few theoretical investigations have addressed the emerging properties from mixed and under- or supersaturated terminations.[14,57]

A number of theoretical investigation have reported on the properties and stability of MXenes terminated by species that are non-inherent to the wet- chemical etching preparation. Despite the apparent influence of the terminating species on the emerging properties, few experimental efforts are underway to tailor the composition of terminating species or even replace the inherent terminations with new and unprecedented ones. This is partly explained by the difficulty to etch MXenes but also the lack of feasible routes for removing the inherent terminations in order to reterminate the MXene.

It is clear that the etchant constitute the first and simplest choice towards tailored terminations and there has been some activity in this field.[36,48,58-60] Further tailoring of the terminating species can also be achieved using post etching treatments such as by $\mathrm{K}^{+}$ion intercalation.[60] Using potassium hydroxide and potassium acetate in aqueous solutions, it was shown that the relative $\mathrm{F}$ termination content is reduced significantly as Ti-F bonds were suggested to become unstable at high $\mathrm{pH} .[10]$ A facile method for entirely avoiding terminations by $\mathrm{F}$, and promoting $\mathrm{O}$ containing species, is by alternatives to $\mathrm{HF}$ or LiF etching. A recent investigation employed a hydrothermal process to etch $\mathrm{Ti}_{3} \mathrm{AlC}_{2}$ at $270{ }^{\circ} \mathrm{C}$ in a $27.5 \mathrm{~m} \mathrm{NaOH}$ solution. The results showed the removal of $\mathrm{Al}$ from $\mathrm{Ti}_{3} \mathrm{AlC}_{2}$ resulting in a multilayer $\mathrm{Ti}_{3} \mathrm{C}_{2} \mathrm{~T}_{\mathrm{x}}$ with $\mathrm{O}$-based terminations and a yield of $92 \mathrm{wt} \%$. [61] 
While showing promise for tuning the terminating species and hence the MXene properties, available terminating species remain limited to those inherent to the etchant. Alcohol or aqueous-alcohol solutions has been proposed to replace the aqueous solution and density-functional tight-binding (DFTB), DFT, and MD calculations have shown that methoxy-terminated MXenes can be stable. These findings suggest MXenes as viable candidates for catalytic applications such as in esterification processes.[62]

An altogether different route for synthesising MXenes, which additionally enables a wide range of novel surface terminations, was recently developed using molten salt, e.g. $\mathrm{ZnCl}_{2}$, to initially substitute the A element and eventually exclusively terminate the emerging MXene surfaces by $\mathrm{Cl}$, by use of excessive $\mathrm{ZnCl}_{2}$.[63,64] This route promises to vastly expand the property space for MXenes, enabling entirely new and unprecedented surface chemistries.

An attractive way of tailoring the surface terminations is by post-etch processing, since this allows for high throughput MXene synthesis through tailored recipes. Initial depeletion of the MXene surfaces from $\mathrm{F}$ terminations, as described above, renders $\mathrm{O}$ the only remaining termination. [45] Subsequent exposure of the F-depleted surfaces to $\mathrm{H}_{2}$ in a heated environment additionally reduces the remaining $\mathrm{O}$ terminations and leaves a depleted MXene surface according to the following reaction:

$\mathrm{Ti}_{3} \mathrm{C}_{2}(\mathrm{O})_{2}+2 \mathrm{H}_{2}=\mathrm{Ti}_{3} \mathrm{C}_{2}+2 \mathrm{H}_{2} \mathrm{O}$

Consequently, a feasible route for reducing the surface terminations exists, and is schematically described and experimentally shown in fig. 5. [65] This approach enable fundamental investigations 
of non-terminated MXenes, and additionally allows for re-termination by non-inherent species such as $\mathrm{CO}_{2}$, see fig. $5 \mathrm{~d}$,h.

Essentially, surface terminations are most easily chosen through the etchant, but with a route that allows for non-inherent terminations, the available surface chemistries increases dramatically and vastly widens the property space for the emerging MXenes.

a) As prepared

b) Heat treatment

c) $\mathrm{H}_{2}$ exposure

d) $\mathrm{CO}_{2}$ exposure

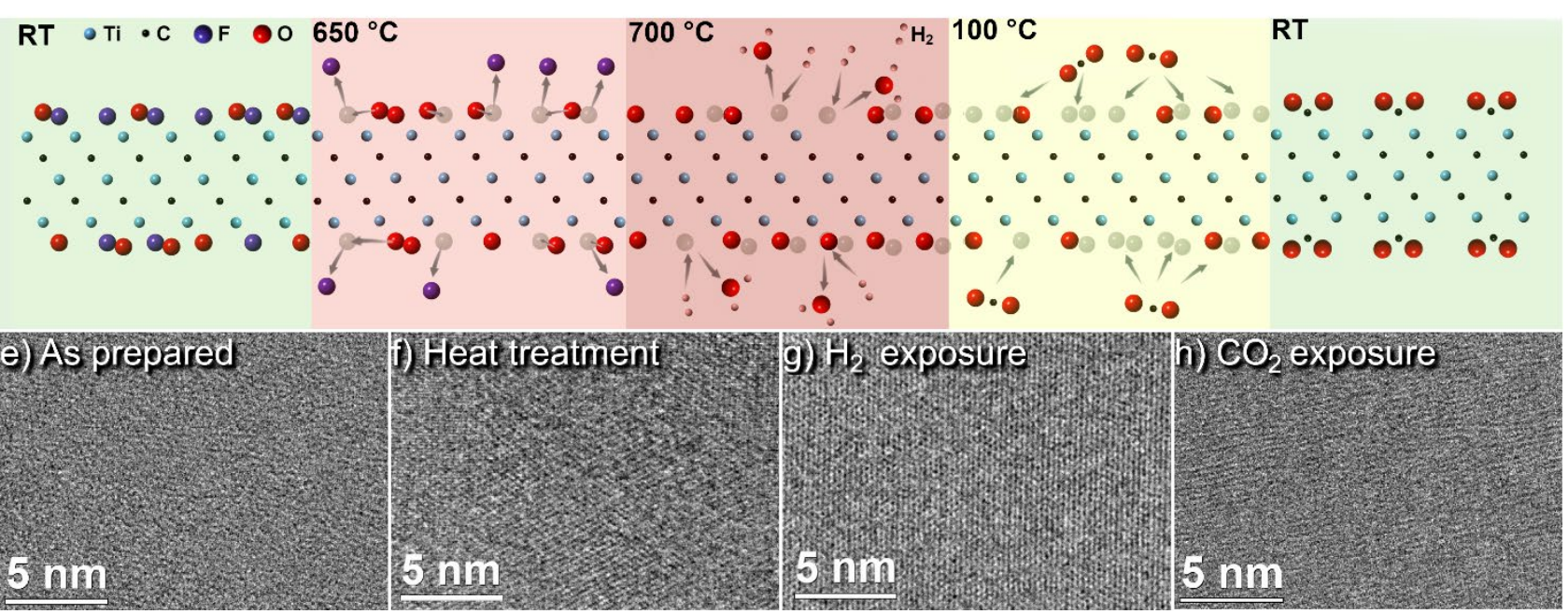

Figure 5: Cross-sectional schematics of a $\mathrm{Ti}_{3} \mathrm{C}_{2} T_{x}$ flake illustrating the processing required to replace the inherent with non-inherent surface terminations a) as prepared, b) heat treatment, c) $\mathrm{H}_{2}$ exposure, and d) $\mathrm{CO}_{2}$ exposure. The corresponding plan-view TEM images after each step are shown in $e^{-h)}$ and were acquired from e) as prepared, f) high temperature treated, g) high temperature $\mathrm{H}_{2}$ etched, and h) low temperature $\mathrm{CO}_{2}$ exposed. Adapted from [65]

Owing to the high volumetric and gravimetric surface area, the majority of manuscripts published on MXenes are currently oriented towards energy storage, where MXenes have reached a level of maturity that renders them feasible for large-scale applications. [9] Obviously, the same prerequisites can be employed in a range of alternative applications such as electromagnetic interference shielding, 
sensing, filtering and water purification, where MXenes have shown a significant capacity.[66-68] The ability to incorporate catalyst elements in the structure, in vacancies, [37,38] or on the surface makes MXene an excellent host for single atom catalysts or for catalyst particles, in addition to the inherent catalytic properties of MXenes that have been proposed for oxygen reduction reaction (ORR), [69] oxygen evolution reaction (OER), [70] hydrogen evolution reaction (HER), [7,71,72] for $\mathrm{CO}$ oxidation and $\mathrm{CO}_{2}$ conversion. [73,74] Moreover, MXenes have been investigated as media for Hydrogen or methane storage. [75,76] Conclusively, catalysis and chemical adsorption make for underexploited applications for MXene, where we predict these Materials to gain momentum in the future as a direct consequence of their significant tailoring potential.

\section{Conclusions}

In this review we have discussed MXenes in their role as a versatile and growing family of 2D materials, predominantly owing to their ability for structural tuning, alloying, and available surface terminations. This extensive set of variables for property tuning, make MXenes an outstanding new class of 2D materials.

In the last few years, a new subclass of the MXene family have emerged in the form of chemically ordered alloy MXenes. The chemical order is displayed both out-of-plane, with alternating layers of single elements in a sandwich structure, or in-plane, with 2 M-elements of a 2:1 composition in a chemically ordered arrangement. These findings have spurred the general notation of $o$-MAX and $i$ MAX for the parent materials, with corresponding notation for the derived MXenes. Importantly, the $i$-MAX phases have also allowed synthesis of MXenes with ordered vacancies. Altogether, these novel laminates have expanded the range of metals that can be incorporated in new MAX phases/ MXenes, such as Sc, Y, and W. As the number of novel phases increases steadily, these ordered 
phases will likely soon make a sizeable part of the known MAX phases and MXenes, and also have an impact on the general 2D materials development.

We also outlined outstanding challenges and opportunities in terms of terminations. This includes insight into the identification of and the stability of surface terminations as well as an understanding of the level of surface saturation with respect to terminating specie. In addition, we have discussed current routes for manipulation of available terminations, both during synthesis through choice of etchant as well as post-etching manipulation through intermediate removal of inherent terminations and subsequent re-termination of the surfaces by non-inherent terminations.

Finally, we envision that MXenes will have a significant impact on future 2D materials research, resulting in unprecedented properties and applications in the years to come. With MXenes, 2D materials science springboards from rigid structures to atom by atom level design of the 2 dimensional sheet. 


\section{Acknowledgements}

The authors acknowledge the Swedish Research Council for funding under Grants Nos. 2016-04412 and 2013-8020. The Knut and Alice Wallenberg's Foundation is acknowledged for support of the electron microscopy laboratory in Linköping, a Fellowship grant and a project grant (KAW 2015.0043). The authors also acknowledge Swedish Foundation for Strategic Research (SSF) through project funding (EM16-0004) and the Research Infrastructure Fellow RIF 14-0074. The authors finally acknowledge support from the Swedish Government Strategic Research Area in Materials Science on Functional Materials at Linköping University (Faculty Grant SFO-Mat-LiU No. 2009 00971). 


\section{References}

[1] M. Naguib, et al. Adv. Mater. 23, 4248 (2011)

[2] M. Naguib, et al. ACS Nano 6, 1322 (2012)

[3] M. Naguib, et al. J. Am. Chem. Soc. 135, 15966 (2013)

[4] M. Khazaei, et al. Adv. Funct. Mater. 23, 2185 (2013)

[5] M. Khazaei, M. Arai, T. Sasaki, M. Estili, Y. Sakka, Phys. Chem. Chem. Phys. 16, 7841 (2014)

[6] M.W. Barsoum 2013 MAX Phases: Properties of Machinable Ternary Carbides and Nitrides (New York: Wiley)

[7] M. Naguib, V.N. Mochalin, M.W. Barsoum, Y. Gogotsi, Adv. Mater. 26, 992 (2014)

[8] B. Manoun, O.D. Leaffer, S. Gupta, E.N. Hoffman, S.K. Saxena, J.E. Spanier, M.W. Barsoum, Solid State Commun., 149, 1978 (2009)

[9] B. Anasori, M. R. Lukatskaya and Y. Gogotsi Nat. Rev. Materials 2, 16098 (2017)

[10] Y. Dall'Agnese, M.R.Lukatskaya, K.M.Cook, P.-L. Taberna, Y. Gogotsi, P. Simon Electrochem. Comm., 48, 118 (2014)

[11] Z. Liu, E. Wu, J. Wang, Y. Qian, H. Xiang, X. Li, Q. Jin, G. Sun, X. Chen, J. Wang, M. Li, Acta Mater., 73, 186 (2014)

[12] B. Anasori, Y. Xie, M. Beidaghi, J. Lu, B.C. Hosler, L. Hultman, P.R. C. Kent, Y. Gogotsi, M.W. Barsoum ACS Nano, 9, 9507 (2015)

[13] R. Meshkian, Q. Tao, M. Dahlqvist, J. Lu, L. Hultman, J. Rosen Acta Materialia 125476 (2017)

[14] B. Anasori, C. Shi, E.J. Moon, Y. Xie, C.A. Voigt, P.R.C. Kent, S.J. May, S.J. Billinge, M.W. Barsoum, Y. Gogotsi, Nanoscale Horizons 1, 227 (2016)

[15] L. Li, Comput. Mater. Sci. 124, 8 (2016)

[16] Z.W. Seh, K.D. Fredrickson, B. Anasori, J. Kibsgaard, A.L. Strickler, M.R. Lukatskaya, Y. Gogotsi, T.F. Jaramillo, A. Vojvodic, ACS Energy Lett., 1, 589 (2016)

[17] A.D. Handoko, K.D. Fredrickson, B. Anasori, K.W. Convey, L.R. Johnson, Y. Gogotsi, A. Vojvodic, Z.W. Seh, ACS Applied Energy Materials 1, 173 (2018) 
[18] H. Kim, B. Anasori, Y. Gogotsi, H.N. Alshareef, Chem. Mater. 29, 6472 (2017)

[19] M. Khazaei, A. Ranjbar, M. Arai, S. Yunoki, Phys. Rev. B 94, 125152 (2016)

[20] J. Yang, X. Zhou, X. Luo, S. Zhang, L. Chen, Appl. Phys. Lett. 109, 203109 (2016)

[21] T.L. Tan, H.M. Jin, M.B. Sullivan, B. Anasori, Y. Gogotsi, ACS Nano 11, 4407 (2017)

[22] Q. Tao, M. Dahlqvist, J. Lu, S. Kota, R. Meshkian, J. Halim, J. Palisaitis, L. Hultman, M.W. Barsoum, P.O.Å. Persson, J. Rosen. Nature communications 8, 14949 (2017)

[23] M. Dahlqvist, J. Lu, R. Meshkian, Q. Tao, L. Hultman, J. Rosen Science Advances 3, e1700642 (2017)

[24] M. Dahlqvist, A. Petruhins, J. Lu, L. Hultman, and J. Rosen, ACS Nano 12, 7761 (2018)

[25] H. Lind, J. Halim, S. Simak, J. Rosén, Phys. Rev. Mater. 1, 044002 (2017)

[26] R. Meshkian, M. Dahlqvist, J. Lu, B. Wickman, J. Halim, J. Thörnberg, Q. Tao, S. Li, S. Intikhab, J. Snyder, M. W. Barsoum, J. Palisaitis, L. Hultman, P.O.Å. Persson, J. Rosen Adv. Mat. 30, 1706409 (2018)

[27] I. Persson', A. el Ghazaly, Q. Tao, J. Halim, S. Kota, V. Darakchieva, J. Palisaitis, M. Barsoum, J. Rosen, P.O.Å. Persson Small 4, 1703676 (2018)

[28] L. Qin, Q. Tao, A. el Ghazaly, J. Fernández-Rodríguez, P. O. Å. Persson, J. Rosen and F. Zhang Adv. Funct. Mater. 28, 1703808 (2018).

[29] M.R. Lukatskaya, S.M. Bak, X. Yu, X.Q. Yang, M.W. Barsoum, Y. Gogotsi, Adv. En. Mater., $\mathbf{5}, 1500589(2015)$

[30] P. Srimuk, J. Halim, J. Lee, Q. Tao, J. Rosen, V. Presser, ACS Sustainable Chemistry \& Engineering, 6, 3739 (2018)

[31] J. Halim, J. Palisaitis, J. Lu, J. Thörnberg, E.J. Moon, M. Precner, P. Eklund, P.O.Å. Persson, M. W. Barsoum, and J. Rosen ACS Appl. Nano Mater., 1, 2455 (2018)

[32] H., Tao, J. Yang, and X. Wang. Phys. Chem. Chem. Phys. 19, 31773 (2017)

[33] L. H. Karlsson, J. Birch, J. Halim, M. W. Barsoum, P. O. Å. Persson, Nano Lett., 15, 4955 (2015) 
[34] X. Sang, Yu Xie, M.-W. Lin, M. Alhabeb, K.L. van Aken, Y. Gogotsi, P.R.C. Kent, K. Xiao, and R.R. Unocic ACS Nano 10, 9193 (2016)

[35] A. Bandyopadhyay, D. Ghosh, and S.K. Pati. Phys. Chem. Chem. Phys. 20, 4012 (2018)

[36] C. E. Ren, M.-Q. Zhao, T. Makaryan, J. Halim, M. Boota, S. Kota, B. Anasori, M.W. Barsoum, and Y. Gogotsi Chem. Electro. Chem., 3, 689 (2016)

[37] J. Zhang, Y. Zhao, X. Guo, C. Chen, C.-L. Dong, R.-S. Liu, C.-P. Han, Y. Li, Y. Gogotsi and G. Wang Nature Catalysis 1, 985 (2018)

[38] D. Zhao, Z. Chen, W. Yang, S. Liu, X. Zhang, Y. Yu, W.-C. Cheong, L. Zheng, F. Ren, G. Ying, X. Cao, D. Wang, Q. Peng, G. Wang, and C. Chen J. Am. Chem. Soc., 141, 4086 (2019)

[39] M. Ashton, K. Mathew, R.G. Hennig, S.B. Sinnott, J. Phys. Chem. C, 120, 3550 (2016)

[40] O. Mashtalir, M. Naguib, V.N. Mochalin, Y. Dall'Agnese, M. Heon, M.W. Barsoum, Y. Gogotsi, Nat. Commun., 4, 1716 (2013)

[41] M.R. Lukatskaya, O. Mashtalir, C.E. Ren, Y. Dall'Agnese, P. Rozier, P.L. Taberna, M. Naguib, P. Simon, M.W. Barsoum, Y. Gogotsi, Science, 341, 1502 (2013)

[42] T. Hu, M. Hu, B. Gao, W. Li, and X. Wang, J. Phys. Chem. C, 122, 18501 (2018)

[43] T. Hu, Z. Li, M. Hu, J. Wang, Q. Hu, Q. Li, and X. Wang J. Phys. Chem. C, 121, 19254 (2017)

[44] J.J. Harris, M. Bugnet, M. Naguib, M.W. Barsoum, G.R. Goward, J. Phys. Chem. C, 119, 13713 (2015)

[45] I. Persson, L.-Å. Näslund, J. Halim, M. W. Barsoum, V. Darakchieva, J. Palisaitis, J. Rosen, P. O. Å. Persson, 2D Mater., 5, 015002 (2017)

[46] L.-Y. Gan, D. Huang, U. Schwingenschlögl, J. Mater. Chem. A 43, 13672 (2013)

[47] M. Naguib, V.N. Mochalin, M.W. Barsoum, Y. Gogotsi, Adv. Mater., 26, 992 (2014)

[48] Q. Tang, Z. Zhou, P. Shen J. Am. Chem. Soc., 134, 16909 (2012)

[49] Y. Xie, P. Kent, Phys. Rev. B, 87, 235441 (2013)

[50] X. Zhang, Z. Ma, X. Zhao, Q. Tang, Z: Zhou, J. Mater. Chem. A, 3, 4960 (2015)

[51] J. Hu, B. Xu, C.Y. Ouyang, Y. Zhang, S. Yang, RSC Adv. 6, 27467 (2016)

[52] D. Magne, V. Mauchamp, S. Célérier, P. Chartier, T. Cabioc’h, Phys. Rev. B 91, 201409 (2015) 
[53] T. Schultz, N.C. Frey, K. Hantanasirisakul, S. Park, S. J. May, V.B. Shenoy, Y. Gogotsi, and

N. Koch DOI: 10.1021/acs.chemmater.9b00414

[54] J. Halim, K. M. Cook, M. Naguib, P. Eklund, Y. Gogotsi, J. Rosen, M. W. Barsoum, Appl. Surf. Sci., 362, 406 (2016)

[55] M.A. Hope, A.C. Forse, K.J. Griffith, M.R. Lukatskaya, M. Ghidiu, Y. Gogotsi and C. P. Grey Phys. Chem. Chem. Phys., 18, 5099 (2016)

[56] J. Palisaitis, I. Persson, J. Halim, J. Rosén and P. O. Å. Persson, Nanoscale, 10, 10850 (2018)

[57] N.M. Caffrey Nanoscale, 10, 13520 (2018)

[58] D. Magne, V. Mauchamp, S. Célerier, P. Chartier, T. Chabioch, Phys. Chem. Chem. Phys, 18, $30946(2016)$

[59] H.-W. Wang, M. Naguib, K. Page, D.J. Wesolowski, Y. Gogotsi, Chem. Mater. 28, 349 (2015)

[60] J. Halim, I. Persson, P. Eklund, P. O. Å. Persson, J. Rosen RSC Advances 8, 36785 (2018)

[61] T. Li, L. Yao, Q. Liu, J. Gu, R. Luo, J. Li, X. Yan, W. Wang, P. Liu, B. Chen, W. Zhang, W. Abbas, R. Naz, and D. Zhang, Angew. Chem., 130, 1 (2018)

[62] A. N. Enyashin, A. L. Ivanovskii, J. Phys. Chem. C, 117, 13637 (2013)

[63] M. Li, J. Lu, K. Luo, Y. Li, K. Chang, K. Chen, J. Zhou, J. Rosen, L. Hultman, P. Eklund, P.O.Å. Persson, S. Du, Z. Chai, Z. Huang, and Q. Huang J. Am. Chem. Soc., 141, 4730 (2019)

[64] J. Lu, I. Persson, H. Lind, M. Li, Y. Li, K. Chen, J. Zhou, S. Du, Z. Chai, Z. Huang, L.

Hultman, J. Rosen, P. Eklund, Q. Huang, P.O.Å. Persson arXiv:1901.05212

[65] I. Persson, J. Halim, H. Lind, T. W. Hansen, J. B. Wagner, L.-Å. Näslund, V. Darakchieva, J.

Palisaitis, J. Rosen and P.O.Å. Persson, Adv. Mater., 31, 1805472 (2019)

[66] F. Shahzad, M. Alhabeb, C.B. Hatter, B. Anasori, S.M. Hong, C.M. Koo, Y. Gogotsi

Science 353, 1137 (2016)

[67] X.-F. Yu, Y.-C. Li, J.-B Cheng, Z.-B. Liu, Q.-Z. Li, W.-Z. Li, X. Yang, and B. Xiao, ACS Appl. Mater. Interfaces 7, 13707 (2015) 
[68] Q. Peng, J. Guo, Q. Zhang, J. Xiang, B. Liu, A. Zhou, R. Liu, and Y. Tian, J. Am. Chem. Soc. 136, $4113(2014)$

[69] Z. Zhang, H. Li, G. Zou, C. Fernandez, B. Liu, Q. Zhang, J. Hu, Q. Peng, ACS Sustainable Chem. Eng., 4, 6763 (2016)

[70] J. Liu, Y. Liu, N. Liu, Y. Han, X. Zhang, H. Huang, Y. Lifshitz, S.-T. Lee, J. Zhong, Z. Kang Science, 347, 970 (2015)

[71] Z.W. Seh, K.D. Fredrickson, B. Anasori, J. Kibsgaard, A.L. Strickler, M.R. Lukatskaya, Y. Gogotsi, T.F. Jaramillo, A. Vojvodic, ACS Energy Lett., 1, 589 (2016)

[72] G. Gao, A.P. O’Mullane, A. Du, ACS Catal., 7, 494 (2017)

[73] X. Zhang, J. Lei, D. Wu, X. Zhao, Y. Jing, Z. Zhou J. Mater. Chem. A, 4, 4871 (2016)

[74] N. Li, X. Chen, W.-J. Ong, D.R. MacFarlane, X. Zhao, A.K. Cheetham, and C. Sun, ACS Nano, 11, 10825 (2017)

[75] X. Li, G. Fan, and C. Zeng, Int. J. Hydrogen Energy, 39, 14927 (2014)

[76] F. Liu, A. Zhou, J. Chen, J. Jia, W. Zhou, L. Wang, and Q. Hu, Appl. Surf. Sci., 416, 781 (2017) 